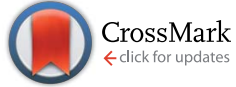

Cite this: Soft Matter, 2014, 10, 8685

Received 8th August 2014

Accepted 9th September 2014

DOI: $10.1039 / c 4 s m 01763 c$

www.rsc.org/softmatter

\section{Experimental and molecular dynamics characterization of dense microemulsion systems: morphology, conductivity and SAXS $\uparrow$}

\begin{abstract}
E. Negro, ${ }^{a}$ R. Latsuzbaia, ${ }^{a}$ A. H. de Vries $^{b}$ and G. J. M. Koper ${ }^{\star a}$
Microemulsions are exciting systems that are promising as tuneable self-assembling templating reaction vessels at the nanoscale. Determination of the nano-structure of microemulsions is, however, not trivial, and there are fundamental questions regarding their design. We were able to reproduce experimental data for an important microemulsion system, sodium-AOT-n-heptane-water, using coarse-grained simulations involving relatively limited computational costs. The simulation allows visualization and deeper investigation of controversial phenomena such as bicontinuity and ion mobility. Simulations were performed using the Martini coarse-grained force field. AOT bonded parameters were fine-tuned by matching the geometry obtained from atomistic simulations. We investigated several compositions with a constant ratio of surfactant to oil while the water content was varied from 10 to $60 \%$ in weight. From mean square displacement calculation of all species, it was possible to quantify caging effects and ion mobility. Average diffusion coefficients were calculated for all charged species and trends in the diffusion coefficients were used to rationalize experimental conductivity data. Especially, the diffusion coefficient of charged species qualitatively matched the variation in conductivity as a function of water content. The scattering function was calculated for the hydrophilic species and up to $40 \%$ water content quantitatively matched the experimental data obtained from small angle X-ray scattering measurements. For higher water contents, discrepancies were observed and attributed to a nearby phase separation. In particular, bicontinuity of water and oil was computationally visualized by plotting the coordinates of hydrophilic beads. Equilibrated coarse-grained simulations were reversed to atomistic models in order both to compare ion mobility and to catch finer simulation details. Especially, it was possible to capture the intimate ion pair interaction between the sodium ion and the surfactant head group.
\end{abstract}

\section{Introduction}

Bicontinuous microemulsions (BMEs) are a special class of thermodynamically stable, single phase emulsions, type IV in the Winsor classification, ${ }^{1}$ where surfactant is mixed with almost equal amounts of oil and water in such a way that both water and oil form continuous channels. Such a structure is stabilized by a large amount of surfactant $(\sim 50 \%$ in weight with respect to the final mixture). The concept of BMEs was introduced in $1976^{2}$ and since then, these colloidal structures have been extensively studied using various techniques. However,

${ }^{a}$ Department of Chemical Engineering, Delft University of Technology, Julianalaan 136, 2628 BL Delft, Netherlands. E-mail: g.j.m.koper@tudelft.nl

${ }^{b}$ Molecular Dynamics Group, Groningen Biomolecular Sciences and Biotechnology Institute and Zernike Institute for Advanced Materials, University of Groningen, Nijenborgh 7, 9747 AG Groningen, Netherlands

$\dagger$ Electronic supplementary information (ESI) available: Simulations details, final snapshots, continuity, morphology, MSD and SAXS analysis additional images. See DOI: $10.1039 / \mathrm{c} 4 \mathrm{sm} 01763 \mathrm{c}$ their potential for application has been largely overlooked in comparison to that of reverse micelles (RMs).

BMEs recently resulted in an optimal template for synthesis of metallic nanoparticles ${ }^{3-6}$ and Metal Organic Framework (MOF) nanocrystals, ${ }^{7}$ for enzymatic catalysis, ${ }^{8}$ and for assembly of mesoporous nanocomposites. ${ }^{9}$ Additionally, they recently attract again great interest for surfactant "Enhanced Oil Recovery" applications having oil scarcity as the main driving force. ${ }^{\mathbf{1 0}}$ BMEs provide a sponge-like mesoporous nanostructure with a large interfacial area between polar and apolar domains. They allow the confinement of species in the water phase as do RMs but they have the advantage that the template motion is itself constrained. For example, these structures are believed to be crucial for the synthesis of stable nanoparticles since they allow the metal precursor ions to diffuse freely but they do not allow the diffusion of nanoparticles. ${ }^{5}$ In that way, the reaction timescale is much faster than the growth timescale, resulting in the formation of monodisperse nanoparticles. Additionally, higher yields can be achieved because of the higher water content (up to $40 \%$ in weight) compared to conventional RM synthesis., ${ }^{\mathbf{4 5}}$ 
Understanding how ME structure depends on composition is crucial for providing a molecular basis for interpreting experimental results and investigating the effects on for example nanoparticles synthetized in them.

Molecular Dynamics (MD) simulations have been widely used to investigate microemulsion systems, especially concerning RM size, shape and shape transitions, ${ }^{11-13}$ water behaviour as a function of the distance from the interface ${ }^{\mathbf{1 4 - 1 9}}$ and head-group-solute interactions..$^{\mathbf{1 8 , 2 0 , 2 1}}$ However, simulations of ternary systems were limited to low surfactant concentrations and deal mainly with RM systems. One of the reasons is that most of the simulations were atomistic, or all atoms (AA), whose computational costs are in general too high to capture timescales and length scales necessary to characterize microemulsion systems containing large amount of surfactant, in which diffusion is slowed down by higher viscosity of the system and characteristic dimensions are on the order of tens of nanometers. ${ }^{22}$

Coarse-grained (CG) simulations allow significant computational cost reduction compared to AA simulations and are suitable to simulate systems requiring microseconds and micrometres. Hybrid systems, using AA for interfacial regions and CG for the rest, have also been adopted to reduce computational costs. ${ }^{22,23}$ The Martini CG force-field (FF), a fast, easy and efficient simulation tool, was developed in 2003 by Marrink et al. ${ }^{24}$ for biomolecular applications, becoming in less than a decade one of the most widely used CG force field for a broad range of applications. In particular it appears well suited to study formation of micelles, allowing simulation times long enough to equilibrate this kind of systems. ${ }^{13,25-27}$

In this work, we aim to investigate dense microemulsion systems formed with dioctyl sodium sulfosuccinate or docusate sodium, known as Na-AOT, because of its widespread use in many applications and long standing investigation in our group as template for metal nanoparticles synthesis. This molecule has been widely investigated by AA MD simulations ${ }^{12,16,18,19,28}$ and more recently by a few CG models. ${ }^{\mathbf{1 0 , 2 9}}$

The goal of this work is to provide a Martini CG model for the system water-Na-AOT- $n$-heptane, to map out an important part of the ternary phase diagram and to compare the resulting structures/morphologies to geometrical models available in the literature $^{\mathbf{3 0}}$ and to experimental characterizations such as conductivity and small angle X-ray scattering (SAXS) data. First, Na-AOT and $n$-heptane bonded interaction parameters are fine tuned in Martini according to AA MD simulations and part of the phase diagram is mapped out and compared to a theoretical model. Bicontinuity is computationally visualized. Secondly, diffusion coefficients of charged species are compared to experimental conductivity data. Mean square displacements (MSDs) of charged species are used to investigate caging effects. Thirdly, scattering functions calculated from radial distribution functions of hydrophilic species are compared to SAXS data. Finally, after back mapping equilibrium structures to the AA model of three CG simulations, further AA MD simulations are performed to evaluate effects due to CG loss of detail compared to AA.

\section{Methods}

\section{Phase diagram calculation - the geometrical model}

A phase diagram of the ternary system employed in this study was calculated using a simplistic geometrical model developed by Andre et al., ${ }^{30}$ that predicts structural transitions and supraaggregation processes which are imposed by geometrical constraints. The model is solely based on a geometry of surfactant and related curvature of the oil-water interface and therefore requires calculations of the: (i) composition of the system, here expressed as the water weight fraction, $\bar{w}$; (ii) surfactant parameter, $s$, which is given by the ratio $v /\left(l_{\mathrm{s}} a_{0}\right)$ where $v$ is the molecular volume of the surfactant, $l_{\mathrm{s}}$ is the effective chain length of the surfactant tail, and $a_{0}$ is the optimal head group surface area; $;^{31,32}$ and (iii) packing parameter, which is the ratio between the volume actually occupied by the cylinders/ spheres and that of the unit cells. ${ }^{30}$ During all calculations it is assumed that all the surfactant is located in the oil-water interface. ${ }^{30}$ Based on the geometrical model, radii of spherical, $R_{\mathrm{w}_{\_} \mathrm{S}}$, and cylindrical, $R_{\mathrm{w}_{-}}$, aggregates can be deduced according to eqn (1) and (2), respectively:

$$
\begin{aligned}
& R_{\mathrm{w} \_\mathrm{s}}=3 \bar{w} s l_{\mathrm{s}} \\
& R_{\mathrm{w} \_\mathrm{c}}=2 \bar{w} s l_{\mathrm{s}}
\end{aligned}
$$

\section{Materials and experimental methods}

The surfactant, sodium bis(2-ethylhexyl)sulphosuccinate, also known as Na-AOT $\left(\mathrm{C}_{20} \mathrm{H}_{37} \mathrm{NaO}_{7} \mathrm{~S}, 99 \%\right)$, and the oil, $n$-heptane (99.9\%), were purchased from Sigma-Aldrich BV and used as received. Water produced by Milli-Q Ultra-Pure-Water purification system of Millipore BV was used in all sample formulations. All preparations and analysis were carried out at room temperature and atmospheric pressure. Surfactant and oil were mixed in the ratio $2: 1$ in weight and sonicated for one hour to speed up dissolution of the surfactant. Subsequently, water was added to the mixture of oil and surfactant in different ratio in weight. After circa 1 hour, the microemulsions were found to be clear and were considered homogeneous and ready for further experiment or analysis. Conductivity measurements were carried out using a conductivity meter model 712 from Metrohm AG. Small angle X-ray scattering (SAXS) was conducted using an AXS D8 Discover instrument from Bruker AG. Microemulsion samples where filled in a $1 \mathrm{~mm}$ thick quartz capillary set at a distance of $30 \mathrm{~cm}$ from the detector. The X-ray source was a tube operated at $40 \mathrm{kV}$ and $40 \mathrm{~mA}$ and produced predominantly copper $\mathrm{K} \alpha$ radiation of wavelength $0.154 \mathrm{~nm}$. The scattering data from the experiments were radially integrated obtaining the intensity as a function of $d$-spacing obtained by applying Bragg's law. Dynamic light scattering (DLS) measurements were performed on the Zetasizer Nano ZS from Malvern Instruments Limited using the $173^{\circ}$ angle noninvasive back-scatter mode and the M3-phase analysis light scattering mode, respectively. The instrument had a red $4.0 \mathrm{~mW}$ $633 \mathrm{~nm} \mathrm{He}-\mathrm{Ne}$ laser. The multiple peak high-resolution fitting 
procedure was used to obtain the particle size distribution from the auto-correlation function.

\section{Computational methods}

All simulations were performed with the GROMACS simulation package version 4 (ref. 33) using periodic boundary conditions in all directions.

Coarse-grained simulations. The CG Martini FF version 2.0 developed by Marrink et al. ${ }^{24}$ was employed to model the present system. This FF uses a basic 4-to-1 mapping scheme of chemical functional groups to single beads. Beads are classified according to their polarity as $\mathrm{Q}$ (charged), $\mathrm{P}$ (polar), $\mathrm{N}$ (neutral), and $\mathrm{C}$ (apolar). Na-AOT was parameterized using 8 beads, a Qd bead with charge +1 for the hydrated $\mathrm{Na}^{+}$-ion, a Qa bead with charge -1 for the surfactant sulphonate anion head, $2 \mathrm{Na}$ beads for the ester groups and $4 \mathrm{C} 1$ beads for the aliphatic chains. The $\mathrm{d}$ and a additions to bead types denote hydrogen bond donor and acceptor capabilities, respectively. $n$-Heptane was parameterized using $2 \mathrm{C} 1$ beads. Groups of 4 water molecules are represented by one polar $\mathrm{P} 4$ bead. The only charged beads in this model are the ones representing the hydrated $\mathrm{Na}^{+}$-ion and the sulphonate anion. Fig. 1 shows the scheme for Na-AOT and $n$ heptane mapping. The bead types determine the strength of the non-bonded interactions according to the interaction matrix published by Marrink et $a l .{ }^{24}$ Non-bonded interactions were modelled by shift functions, which cause the distance-dependent potentials and forces to smoothly go to zero at the cut-off distance instead of showing a discontinuity there. A cut-off of $1.2 \mathrm{~nm}$ was employed for all non-bonded interactions. The Lennard-Jones (LJ) potential was smoothly shifted to zero between 0.9 and $1.2 \mathrm{~nm}$. A similar approach was employed for the Coulomb potential with a relative permittivity of 15 and a shift function from 0 to $1.2 \mathrm{~nm}$.

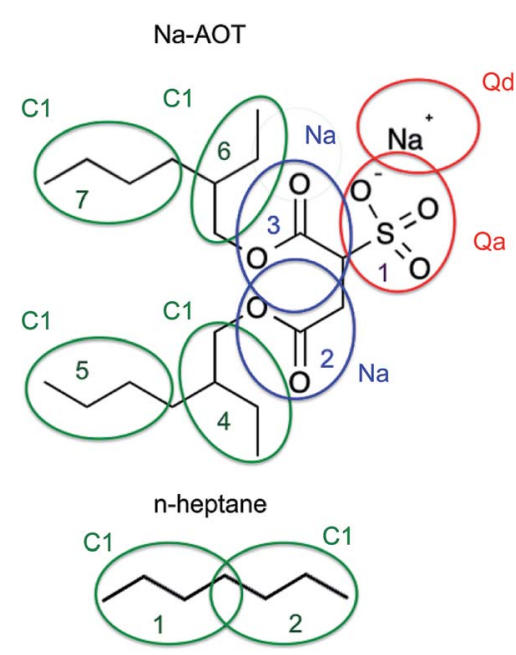

Fig. 1 The coarse-grained model for Na-AOT surfactant and $n$ heptane. Circles include the atoms that are grouped into beads and are coloured to indicate their nature: red- $Q$, blue- $N$, green- $C$. The numbers reflect the order in which the model is represented in the topology.
CG systems were prepared by first randomly placing 100 AOT molecules in a cubic simulation box of dimensions $x y z \mathrm{~nm}$; values are specified for each simulation in Table 2 and S1-S3. $\dagger$ Next, $222 n$-heptane molecules were added randomly to the same volume, and finally, the volume was filled with randomly placed ion and water beads. Each system was energy minimized using the steepest descent method for 500 steps. These systems were then replicated once in each dimension, creating a simulation volume 8 times larger than the initial set-up. After a short MD relaxation run, simulations of $1 \mu$ s were performed, allowing the system to self-assemble and equilibrate. Analysis of morphology, diffusion, and structural characterization was performed on the final $500 \mathrm{~ns}$ of the simulation. All simulation times reported here are unscaled (see also discussion of diffusion results). The initial velocities were randomly assigned from a Maxwell distribution at the reference temperature. The equations of motion were integrated numerically using a $20 \mathrm{fs}$ time step. Water, surfactant molecules, ions and oil were separately coupled to a Berendsen thermostat at $298 \mathrm{~K}$ with a common coupling time of $1 \mathrm{ps}^{34}$ For CG simulations containing 10,15 and $60 \%$ water, the pressure was isotropically controlled at 1 bar using a Berendsen barostat with a coupling time of 3 ps with an isothermal compressibility of $3 \times 10^{-5} \mathrm{bar}^{-1}$. For simulations containing $10,25,30,35,40,45,50$ and $55 \%$ water, the pressure was anisotropically controlled at 1 bar using a Berendsen barostat with a coupling time of 3 ps and with an isothermal compressibility of $3 \times 10^{-5} \mathrm{bar}^{-1}$ in $x, y, z$ directions and off-diagonal compressibility set to 0 in order to keep the box rectangular.

Bonded parameter fine-tuning based on AA simulations. AA simulations (see below) were carried out to gain more insight into the atomistic details of the structure and to assess how the morphology obtained at CG level behaves at AA level, and initially also in order to refine angle and bond parameters for the bonded interactions between the beads, a procedure that is regularly used in building Martini models, see e.g. ${ }^{35,36} \mathrm{We}$ chose as the reference composition the one containing $20 \%$ water, that according to previous experimental studies gives rise to a bicontinuous phase. ${ }^{3}$ Angle and bond length distributions were calculated for a representative sample of AOT and $n$-heptane molecules after mapping the AA structures from an equilibrated $100 \mathrm{~ns}$ simulation to the CG structure. Mapping was done according to the scheme shown in Fig. 1, and based on calculation of the centre of mass of the (united) atoms constituting a CG bead. The same distributions were calculated for a CG simulation using default Martini FF parameter values, here referred to as standard CG. A number of bond lengths and angles were found to be significantly different between AA and standard CG simulations, and these were adjusted in a new topology, here referred to as either refined CG or CG model.

Atomistic simulations. AA simulations were performed using a united-atom FF, parameter files available upon request, based on GROMOS53A6. ${ }^{37}$ The SPC water model ${ }^{38}$ was used. All bonds were constrained using the Lincs $^{39}$ algorithm, while water was treated as a rigid molecule using the SETTLE ${ }^{40}$ algorithm. A non-bonded cut-off of $1.4 \mathrm{~nm}$ was used for $\mathrm{LJ}$ and Coulomb interactions. Whereas the LJ potential employs a 
straight cut-off, Coulomb interactions were smoothly scaled to zero at the cut-off distance by using the reaction field method due to Tironi et al. ${ }^{41}$ with a relative dielectric constant of 62 . Non-bonded interactions within $0.9 \mathrm{~nm}$ were calculated each step, and interactions between 0.9 and $1.4 \mathrm{~nm}$ were calculated every 10 steps together with an update of the neighbour list and assumed constant in between neighbour list updates. A timestep of $2 \mathrm{fs}$ was used to integrate the equations of motion, which were coupled to a Berendsen barostat ${ }^{34}$ with a compressibility of $4.6 \times 10^{-5} \mathrm{bar}^{-1}$ and a coupling time of $0.5 \mathrm{ps}$, as well as to a Berendsen thermostat at the reference temperature of $298 \mathrm{~K}$ and a coupling time of $0.1 \mathrm{ps}$. Periodic boundary conditions were used, either in a cubic or rectangular box, similar to the CG set-ups (see above). Water and ions formed one temperature group separate from a temperature group containing the AOT and $n$-heptane.

Back mapping methods. Resolution changes between AA and CG representations were achieved using the methods due to Rzepiela et al., ${ }^{\mathbf{4 2}}$ which requires a special version of GROMACS, for the fine-tuning of CG parameters and the more recent one due to Wassenaar et al. ${ }^{\mathbf{4 3}}$ which is computationally more efficient and more user friendly, for the diffusion coefficients and morphology investigation. AA structures were mapped to CG structures by calculating the centre of mass of the (united) atoms assigned to their respective beads, according to the scheme shown in Fig. 1. Thus, CG positions are uniquely defined in terms of AA positions. The AA positions are not uniquely defined by the CG positions. Back mapping procedures assign initial positions to AA particles based on the CG positions, and then try to relax the initial structure to a relevant and reasonable AA structure compliant with the AA FF. The method due to Rzepiela et al. assigns the AA positions associated with a particular CG bead randomly within a sphere around the CG bead position, and then anneals the structure, starting at high temperature and with a modified AA FF in which large forces due to unfavourable contacts are capped to a maximum value. The method due to Wassenaar et al. allows for a more controlled reconstruction of AA positions based on CG bead positions in which the connectivity between CG beads may be used to place AA particles already in correct orientations. In our experience, this second method usually is more stable and requires less computational effort in obtaining a reasonable starting structure for subsequent MD simulations. In back mapping, we replaced a water bead by four water molecules and a $\mathrm{Na}^{+}$bead by a $\mathrm{Na}^{+}$ion and three water molecules.

\section{Analysis}

Morphology and continuity investigation. The overall morphology was determined by visual inspection using the program VMD. ${ }^{44}$ Colouring the different types of components differently, and rendering a surface around the water beads establishes the morphology. The morphology was classified as one of the following. If the structure contains reverse micelles or interconnected reverse micelles (water spheres surrounded by surfactant) the morphologies are denoted by RM and IRM, respectively. Worm-like and interconnected worm like structures are denoted $\mathrm{W}$ and IW, respectively, and bicontinuous structures (water and oil are both continuous throughout the system) are classified as BME. In case of cylinders, cylinders hexagonally packed, cylinders of 2 different sizes (water channels are continuous in one dimension, but otherwise not connected), the morphologies are denoted $\mathrm{C}, \mathrm{HC}$ and $\mathrm{BC}$, respectively. Lamellar structures (water and oil are both continuous in two dimensions, but stacked in a third) are denoted by L, interconnected lamellar structures (water and oil are both continuous in two dimensions and at least one is interconnected in the third) IL. Finally, spherical structures (large water spheres, RM, separated from each other by a thin surfactant and oil layer) can be stacked in body-centered cubic, BCC, or face centred cubic, FCC, pattern, and oil in water micelles (oil spheres surrounded by surfactant) are denoted $\mathbf{M}$. Combinations of these morphologies are possible. While visual inspection using VMD provides some insight into the morphology of the structure, the continuity of certain components may be seen more clearly by simple plots showing the beads in a number of slices through the simulation box. Such plots can also be used to estimate the size of compartments and/or channels. Here, we represented the selected beads by a circle with a diameter of $0.5 \mathrm{~nm}$. The box was divided in slices of $1 \mathrm{~nm}$ thickness, and beads in different slices were given a different colour. The combined plots showing the slices in the $x y, y z$, and $x z$ planes provide a rapid insight into the connectedness of the component of interest. Most of these plots are shown in the ESI. $\dagger$ Continuity in $x, y, z$ direction can also investigated by plotting the probability profile to find hydrophilic beads (surfactant head group, $\mathrm{Na}^{+}$and water) or hydrophobic beads (surfactant tails and $n$-heptane) across a cell dimension. Such plots give a rapid idea of the continuity of the two phases and the dimensions of the domains. Most of them are reported in the ESI. $\dagger$

Mean square displacement and diffusion coefficient. The mean square displacement (MSD) was calculated by the built-in GROMACS tool g_msd in order to estimate the spatial extent of random motion for water beads, $\mathrm{Na}^{+}$beads, AOT group of beads and $n$-heptane group of beads. The software calculates the average directional $\mathrm{MSD}_{x}, \mathrm{MSD}_{y}$ and $\mathrm{MSD}_{z}$, according to eqn (3)

$$
\operatorname{MSD}_{k}(t)=\left\langle\left(k_{\tau+t}-k_{\tau}\right)^{2}\right\rangle_{\tau, N}
$$

where $k=x, y, z$, time $t$ is from 0 up to $250 \mathrm{ns,} \tau$ is the reference time, and sample size $N$ is 100 in this work. The MSDs of individual particles are also retained.

The total MSD is the sum of the contributions in different directions according to eqn (4):

$$
\operatorname{MSD}(t)=\operatorname{MSD}_{x}(t)+\operatorname{MSD}_{y}(t)+\operatorname{MSD}_{z}(t)
$$

From the total MSD, an average diffusion coefficient is calculated from the Einstein relation, eqn (5):

$$
D(t)=\frac{\operatorname{MSD}(t)}{6 t}
$$


The combined plots showing the single MSD curves in direction $x, y, z$ provide a rapid insight into the connectedness of the component of interest, discriminating caging effects and free diffusion. Most of these plots are shown in ESI. $\dagger$

Scattering function. We wanted to calculate the SAXS patterns, typically represented as scattered intensity as a function of the magnitude $q$ of the scattering vector $Q$ expressed according to eqn (6):

$$
q=\frac{4 \pi \sin (\theta)}{\lambda}
$$

where $\theta$ is the angle between the incident X-ray beam and the detector measuring the scattered intensity, $I$, and $\lambda$ is the wavelength of the X-rays. ${ }^{45}$

Neglecting inelastic scattering, the scattered intensity, $I$, is proportional to the differential cross section, a measure of the fraction of incident particles that emerge in various directions, that is proportional to the sum of two factors according to eqn (7):

$$
I \propto\left(\frac{\mathrm{d} \sigma}{\mathrm{d} \Omega}\right)_{e l} \propto S_{1}(Q)+S_{2}(Q)
$$

where the first term, $S_{1}(Q)$, depends purely on the scattering properties of the individual atoms and is of little interest for the present study. ${ }^{45}$ Structural information is contained in $S_{2}(Q)$, that depends on the relative positions of different pair of atoms. In case of spherical symmetry, as it happens for disordered liquids or amorphous materials, $S_{2}(Q)$ can be expressed according to eqn (8):

$$
S_{2}(Q)=1+\frac{4 \pi \alpha(Q)}{Q} \int_{0}^{\infty} r[g(r)-1] \sin (Q r) \mathrm{d} r
$$

where $\alpha$ is a coefficient dependent on the average scattering length of the species and their densities and $g(r)$ is the radial distribution function, RDF, of the species. ${ }^{45}$

The built-in GROMACS tools are capable to calculate a scattering function $h(q)$ that is very similar to $S_{2}$, according to eqn (9):

$$
h(q)=4 \pi \rho \int r[g(r)-1] \frac{\sin (Q r)}{Q} \mathrm{~d} r
$$

where $\rho$ is the particle density. ${ }^{45}$

$h(q)$ was calculated using two approaches: simplified and exact. For the simplified approach, RDF was calculated for hydrophilic species $(\mathrm{H})$ only, being water, $\mathrm{Na}^{+}$and head-group beads, according to eqn (10):

$$
g_{\mathrm{HH}}(r)=\frac{V}{4 \pi r^{2} \mathrm{~d} r N_{\mathrm{H}}} \sum_{i \in \mathrm{H}} \sum_{j \in \mathrm{H}} P_{i j}(r)
$$

where $P_{i j}(r)$ is the number of pairs of beads that are at a distance between $r$ and $r+\mathrm{d} r$ from each other, and $N_{\mathrm{H}}$ is the number of particles of type $\mathrm{H}$ in total volume $V$.

For the exact approach, all contributions have been taken into account. In general, for a material consisting of different types of atoms, $g(r)$ can be decomposed into a linear combination of $M(M+1) / 2$ partial correlation functions, $g_{J K}(r)$ according to eqn (11)

$$
g(r)=\sum_{J=1}^{M} \sum_{K=J}^{M} \gamma_{J K} g_{J K}(r)
$$

where $\gamma_{J K}$ is given by eqn (12)

$$
\gamma_{J K} \propto\left(2-\delta_{J K}\right) c_{J} c_{K}\left\langle b_{J}\right\rangle\left\langle b_{k}\right\rangle
$$

which is proportional to $c_{J}$, the concentration of the species $J$ in the sample, and $b_{J}$, the scattering length of the species $J$ which is equal to the number of electrons. The Kronecker delta, $\delta_{J K}$, equals 1 if $J=K$ and 0 otherwise. ${ }^{45}$ The weights are normalized so that:

$$
\sum_{J=1}^{M} \sum_{K=J}^{M} \gamma_{J K}=1
$$

\section{Results and discussion}

\section{Phase diagram investigation}

Theoretical calculation. Fig. 2 shows the phase diagram for the system of interest, water-Na-AOT- $n$-heptane, calculated according to a geometrical model proposed by Andre et al. ${ }^{30}$ for a similar system, water-Cu(AOT) $)_{2}$-isooctane. Compositions were adapted to the differences in molecular mass, while the surfactant parameter used was the same, since for that model a value corresponding to experimentally measured surfactant parameter for Na-AOT was used. Lines on the diagram represent limiting compositions for all possible packings of the colloidal domains in the microemulsion. This diagram is not valid at the lower corners, where phase separation takes place. ${ }^{30}$ In this study, we investigated both experimentally and computationally, the structures corresponding to the compositions a-m depicted in Fig. 2, lying on the line of constant surfactant to oil

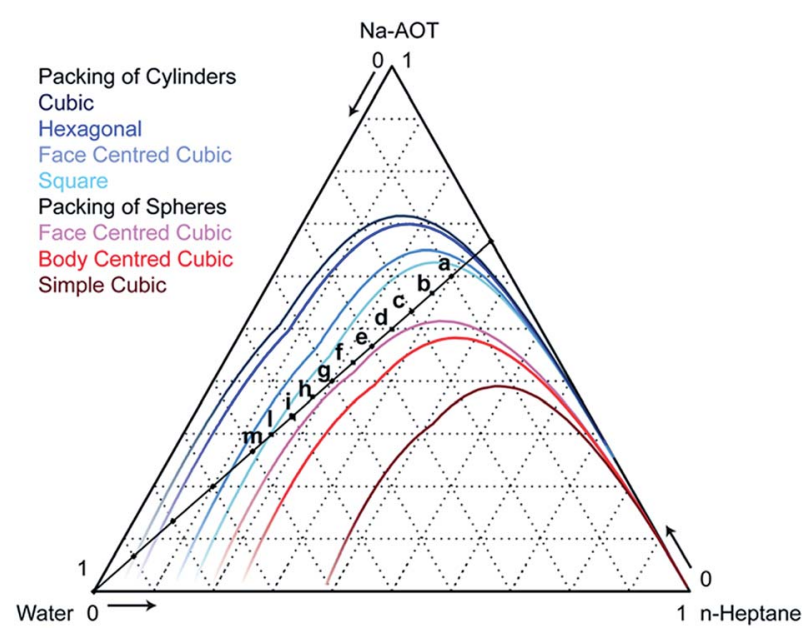

Fig. 2 Phase diagram of water-Na-AOT- $n$-heptane systems calculated according to the geometrical model of Andre. ${ }^{29}$ The coloured lines are the upper boundaries of the region in which the indicated morphology is geometrically allowed. Compositions are expressed in weight fractions. The compositions a through $\mathrm{m}$ are studied experimentally and by modelling. 
weight ratio, equal to 2 . From point a to point $\mathrm{m}$, water content is increased from $10 \%$ to $60 \%$ in weight. We chose that line since it crosses phase diagram regions that were recognized as "bicontinuous" or "interconnected" by previous studies., ,5,30,46,47 $^{3}$

\section{CG Martini simulations}

Refining bonded parameters. The AOT molecule can be coarsegrained using a Martini model 4-to-1 mapping scheme as shown in Fig. 1, where the assignment of the beads is straightforward when comparing the chemical entities sulfonic anion head group, ester linker, and aliphatic tails to those found in the phospholipids for which the model was originally developed. ${ }^{24,48}$ Our initial model employed standard bonded parameters taken from the lipids that are given in Table 1. Also, heptane was modelled using the standard model for octane, a 2bead hydrocarbon. The $\mathrm{Na}^{+}$bead in the Martini model represents a hydrated ion, with 3 or 4 water molecules included in the bead. Here, in calculating the mass ratios of the mixtures, we take the $\mathrm{Na}^{+}$-bead to be associated with three water molecules.

Using spontaneous aggregation from a starting structure containing 80 weight percent Na-AOT and $n$-heptane in $2: 1 \mathrm{w} / \mathrm{w}$ ratio (800 Na-AOT and $1776 n$-heptane molecules) and 20 weight percent water, a predominantly lamellar structure formed within 100 ns, as shown in Fig. 3a. Cell size and number of molecules are reported in Table $S 1, \dagger$ and further analysis of the structural organization in Fig. S1. $\dagger$ It can be clearly seen from Fig. S1 $\uparrow$ that hydrophobic beads are continuous in the $x$ and $y$

Table 1 Bonded parameters: bond lengths, $b_{0}$ corresponding to the length where the force is zero, and force constants, $k_{\mathrm{b}}$; bond angles, $\varphi_{0}$ corresponding to the angle where the force is zero, and force constants, $k_{\varphi}$, for Standard CG and Rrefined CG AOT and $n$-heptane, according to the mapping depicted in Fig. 1

AOT

Standard CG

Bond $\quad b_{0} \mathrm{~nm}^{-1} \quad k_{\mathrm{b}} \mathrm{kJ}^{-1} \mathrm{~mol}^{-1} \mathrm{~nm}^{-2} \quad b_{0} \mathrm{~nm}^{-1} \quad k_{\mathrm{b}} \mathrm{kJ}^{-1} \mathrm{~mol}^{-1} \mathrm{~nm}^{-2}$

\begin{tabular}{lllll}
$1-2$ & 0.47 & 1250 & 0.28 & 1250 \\
$2-3$ & 0.37 & 1250 & 0.39 & 1250 \\
$2-4$ & 0.47 & 1250 & 0.39 & 1250 \\
$4-5$ & 0.47 & 1250 & 0.39 & 1250 \\
$3-6$ & 0.47 & 1250 & 0.39 & 1250 \\
$6-7$ & 0.47 & 1250 & 0.39 & 1250 \\
\hline Angle & $\varphi_{0}{ }^{\circ}$ & \multirow{2}{*}{$k_{\varphi} \mathrm{kJ}^{-1} \mathrm{~mol}^{-1} \mathrm{~nm}^{-2}$} & $\varphi_{0} /{ }^{\circ}$ & \multicolumn{2}{l}{$k_{\varphi} \mathrm{kJ}^{-1} \mathrm{~mol}^{-1} \mathrm{~nm}^{-2}$} \\
\hline \multirow{2}{*}{$1-2-3$} & 120 & 25 & 60 & 45 \\
$1-2-4$ & 180 & 25 & 120 & 25 \\
$3-2-4$ & - & - & 150 & 25 \\
$2-3-6$ & - & - & 130 & 45 \\
$2-4-5$ & 180 & 25 & 100 & 45 \\
$3-6-7$ & 180 & 25 & 100 & 65 \\
\hline
\end{tabular}

HEP

Standard CG

Bond $\quad b_{0} \mathrm{~nm}^{-1} \quad k_{\mathrm{b}} \mathrm{kJ}^{-1} \mathrm{~mol}^{-1} \mathrm{~nm}^{-2} \quad b_{0} \mathrm{~nm}^{-1} \quad k_{\mathrm{b}} \mathrm{kJ}^{-1} \mathrm{~mol}^{-1} \mathrm{~nm}^{-2}$

$\begin{array}{lllll}1-2 & 0.47 & 1250 & 0.35 & 1250\end{array}$

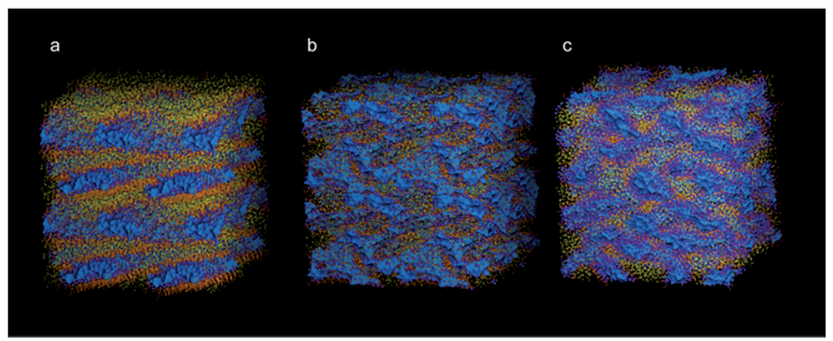

Fig. 3 Final snapshot for the system containing 20\% water for (a) standard CG, $1 \mu \mathrm{s}$, (b) AA, 400 ns, (c) refined CG, $1 \mu \mathrm{s}$. Water - light blue, $n$-heptane - yellow, $\mathrm{Na}^{+}$- blue, AOT head group - purple, AOT tail - orange. The simulated cell has been replicated once in each dimension, creating a simulation volume 8 times larger, in order to allow a better visualization of the structure.

directions but they are not in the $z$ direction. Based on experimental data and the theoretical hard-sphere model, a lamellar morphology is unlikely, and we sought to refine the CG model to better and more specifically represent the AOT and $n$-heptane molecules. Note that the 4-to-1 mapping scheme necessarily makes the model somewhat generic in the sense that using two beads for the lipid tail may represent anything between a 6- and 10-carbon aliphatic chain. In fact, given the parameterization to linear alkanes, the standard CG model may be more representative of a straight-chain Na-di(C8:0)phosphatidic acid-octanewater mixture. Short-chain phosphatidic acids are known to form micelles in water, ${ }^{\mathbf{4 9}}$ and the general effect of adding alkanes to lipids is to promote more inverse curvature assemblies; ${ }^{50}$ the lamellar structure is consistent with these data.

Refinement of the AOT and $n$-heptane models was based on AA simulations using an in-house version of the GROMOS53A6 FF, parameter files available upon request. The same 20/80 water/surfactant + oil w/w mixture as discussed above, containing 800 Na-AOT molecules, formed a BME spontaneously from a random starting structure using this atomistic model, see Fig. 3b. Bond length and angle distributions were measured for the surfactant and $n$-heptane molecules after applying the mapping scheme shown in Fig. 1 to the AA trajectory. We changed reference bond lengths and angles in the CG model according to the peaks of the mapped model distributions, and increased selected force constants for the angles to better reflect the width of the distributions. The parameters are given in Table 1. Although bond distributions tended to be narrower in the mapped model than in the Martini model, we chose to keep the standard bond force constants. An example comparing the distributions found in the mapped AA simulations, in the standard model, and in the refined model is shown in Fig. 4. All comparisons of bond angles, bond lengths and dihedral angles distributions are shown in Fig. $\mathrm{S} 2-\mathrm{S} 4, \dagger$ respectively, while two examples are shown in Fig. 4. Note that in some cases, the mapped distributions are bimodal, e.g. Fig. 4a; these cannot be reproduced using simple harmonic potentials. The main effect of the refined bonded parameters is a better representation of the shape of the AOT molecule, doing justice to the branching of the aliphatic tails and the position of the charged head group. Since it was our aim to stay close to the original Martini model, 
a

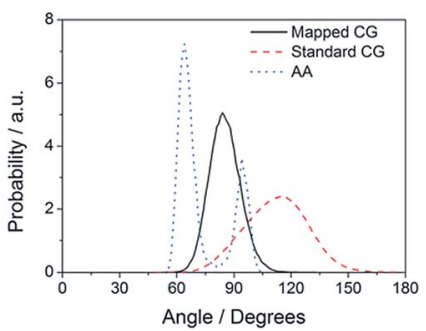

b

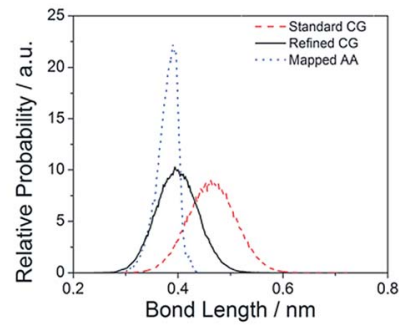

Fig. 4 (a) Bond angle 1-2-3 distribution and (b) bond length 2-4 distribution for CG standard, CG refined and mapped AA. The indices refer to AOT beads depicted in Fig. 1.

no further refinements were made, but it is clear that a closer match could be achieved.

Spontaneous assembly simulations using the refined CG model, in which the bonded parameters represent the shape and size of the Na-AOT and $n$-heptane molecules more specifically, does lead to BME for the 20/80 mixture, see Fig. 3c. Fig. 5 and $\mathrm{S} 5 \dagger$ show that hydrophilic beads form continuous domains in all directions for refined CG and AA simulations, respectively. Thus, at this composition, the AA and CG models are in mutual agreement and consistent with previous interpretations of experimental data and the hard-sphere model.

Phase diagram investigation. We next investigated the behaviour of the CG model along the dilution line shown in Fig. 2, varying the water content from 10 to 60 weight percent, keeping the surfactant-oil weight ratio constant at $2: 1$, corresponding to systems a-m. Systems were formed using spontaneous aggregation simulations starting from random mixtures of surfactant, oil, and water. The compositions, unit cell dimensions, and morphological classifications of the systems are given in Table 2. Fig. 6 shows the final structures of our simulations for the systems.

The morphology of some structures is clearly recognizable. Fig. $6 \mathrm{a}-\mathrm{c}$ corresponding to 10,15 and $20 \%$ water content,

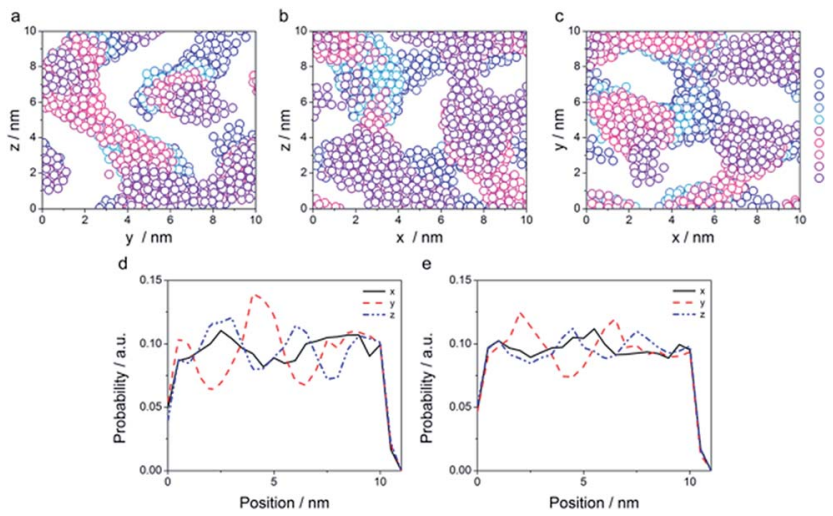

Fig. 5 Slices of the final frame of system c, containing 20\% water, CG refined simulation of planes perpendicular to (a) $x$ axis, (b) $y$ axis, (c) $z$ axis. Only hydrophilic beads are represented, being water, $\mathrm{Na}^{+}$and AOT head-group. Beads are coloured according to the layer they are in. The layers are $1 \mathrm{~nm}$ thick. Distribution profile across Cartesian direction for (d) hydrophilic beads, water, $\mathrm{Na}^{+}$and AOT head group, (e) hydrophobic beads, AOT apolar tail and $n$-heptane.

respectively, show percolating water in a continuous oil phase. According to the phase diagram in Fig. 2, we are below the lines of cylinders that can be packed in all different ways. Due to the large amount of surfactant that can stabilize large water-oil interfaces, the most favourable configuration is the formation of continuous nano-channels of one into the other. Fig. S6, S7† and 5 show that hydrophilic beads form a continuous domain in all directions, and the probability distribution plots show that both hydrophobic and hydrophilic beads are continuous in all directions. In contrast, Fig. 6d (25\% water content) shows cylinders packed in a hexagonal manner, Fig. $6 \mathrm{f}(35 \%)$ spherical micelles packed in a BCC configuration, while Fig. 6e (30\%) a transition between the two. Fig. $6 \mathrm{~g}(40 \%)$ shows spheres arranged in a FCC configuration, Fig. $6 \mathrm{~h}(45 \%)$ worms and Fig. 6i $(50 \%)$ cylinders. In Fig. 61 (55\%), the coexistence of cylinders of two different sizes can be observed, while in Fig. $6 \mathrm{~m}$ $(60 \%)$ spheres are arranged in a BCC configuration. According to phase diagram of Fig. 2, this composition should correspond to cylinders. Experimentally, we observed that this system was tremendously viscous (more than $10 \mathrm{cP}$ ) as discussed in more detail later on when discussing SAXS results. All simulations were started from randomly distributed molecules, to avoid hysteresis phenomena. However, for the system containing $60 \%$ water, due to the high viscosity, we carried out an additional simulation, $\mathrm{m}^{*}$ in Table 2, starting from the final snapshot of the simulation containing $55 \%$ water and adding $5 \%$ water. The system was then enlarged 8 times and simulations were run for $1 \mu \mathrm{s}$. Fig. 6m* shows that in that case, cylinders of two dimensions are obtained. This is likely to be the more realistic configuration, as described later in the conductivity experiments. Additionally, small simulation systems are incapable to simulate proper phase separation, and $60 \%$ water content is at the boundary with phase separation occurring at $65 \%$ water content. Continuity investigations for all simulated systems are reported Fig. S6-S16; $\dagger$ their interpretation in terms of the morphology, and corresponding qualification are reported in Table 2.

It must be observed than when using the standard CG model, see Table $\mathrm{S} 2 \uparrow$ for simulations details, completely different structures were obtained and no BME was found, Fig. S17. $\dagger$ This is due to a bigger volume of the Na-AOT and $n$ heptane molecule and thus of the apolar phase, as proven by the bigger box dimensions, Table S2.† Also the AOT surfactant parameter can be slightly different before and after refinement.

To further characterize the relation with the morphology and macroscopic measurements, a number of further analyses have been carried out. Results are presented in the following sections.

\section{Conductivity}

Experimental results. The conductivity measurements were performed with a conductivity meter. The measured conductivities as function of the water content are shown in Fig. 7a. They can be explained by taking into account two different contributions: the structure, and thus the increase in the conductive cross section, and the conductivity of the aqueous 
Table 2 The compositions, where $n_{\mathrm{w}}, n_{\mathrm{s}}$ and $n_{\circ}$ are the number of water, surfactant and oil molecules, respectively, box dimensions, water continuity, and morphological classifications of the systems corresponding to compositions a-m depicted in Fig. 2

\begin{tabular}{|c|c|c|c|c|c|c|c|}
\hline & Water\% & Box size $\mathrm{nm}^{-3}$ & $n_{\mathrm{w}}$ & $n_{\mathrm{s}}$ & $n_{\mathrm{o}}$ & Continuity $^{c}$ & Structure \\
\hline $\mathrm{a}$ & 10 & $14 \times 8 \times 9$ & 3296 & 800 & 1776 & $X Y Z$ & IW \\
\hline $\mathrm{b}$ & $15^{a}$ & $10 \times 10 \times 10$ & 5216 & 800 & 1776 & $X Y Z$ & BME \\
\hline d & 25 & $11 \times 9 \times 12$ & 9888 & 800 & 1776 & $Z$ & $\mathrm{HC}$ \\
\hline e & 30 & $11 \times 10 \times 11$ & 12704 & 800 & 1776 & $Y Z$ & IW \\
\hline$f$ & 35 & $8 \times 10 \times 16$ & 15968 & 800 & 1776 & $X$ & IM/BCC \\
\hline $\mathrm{i}$ & 50 & $12 \times 12 \times 12$ & 29632 & 800 & 1776 & $Y$ & $\mathrm{C}$ \\
\hline 1 & 55 & $14 \times 9 \times 15$ & 36224 & 800 & 1776 & $Y$ & $\mathrm{BC}$ \\
\hline $\mathrm{m}$ & $60^{a}$ & $26 \times 26 \times 26$ & 355584 & 6400 & 14208 & - & BCC \\
\hline $\mathrm{m}^{*}$ & $60^{b}$ & $34 \times 15 \times 35$ & 355584 & 6400 & 14208 & $Y$ & $\mathrm{BC}$ \\
\hline
\end{tabular}

${ }^{a}$ Isotropic pressure coupling. ${ }^{b}$ Anisotropic pressure coupling, and started after adding $5 \%$ water to the $55 \%$ system (multiplied 8 times and relaxed). ${ }^{c}$ Directions in which water is continuous.

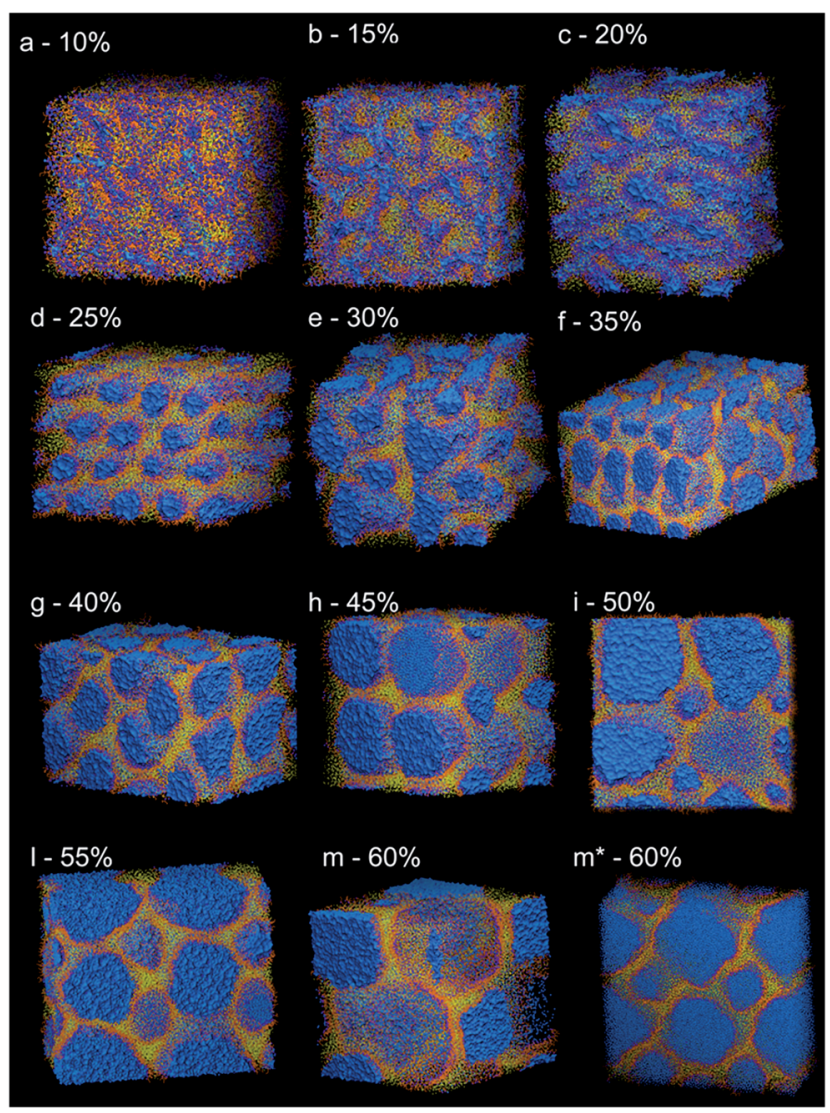

Fig. 6 Snapshot at the end of $1 \mu$ s simulations for the system waterNa-AOT- $n$-heptane using the refined CG model. Water - light blue, $n$-heptane - yellow, $\mathrm{Na}^{+}$- blue, AOT head group - purple, AOT tail orange. Compositions corresponding to the points depicted in Fig. 2. Simulation details are reported in Table 2.

phase itself, dramatically increased by the presence of sodium ions. The conductivity of the water phase was theoretically calculated according to eqn (14) by assuming that all the surfactant cations $\left(\mathrm{Na}^{+}\right)$were contributing to the charge conduction, while the anions were assumed fixed and not contributing:

$$
k=\Lambda_{\mathrm{m}} c
$$

where $k$ is the conductivity in $\mathrm{mS} \mathrm{cm}^{-1}, c$ is the cation concentration in mol $\mathrm{L}^{-1}$ and $\Lambda_{\mathrm{m}}$ is the limiting ionic conductivity in water of $\mathrm{Na}^{+}$at $298 \mathrm{~K}$. Non-idealities such as the decrease in the ionic conductivity according to Kohlrausch's law are neglected.

For the microemulsion of water content below 10\%, the conductivity is very low and does not vary much. At these very low water contents microemulsion structures are formed in

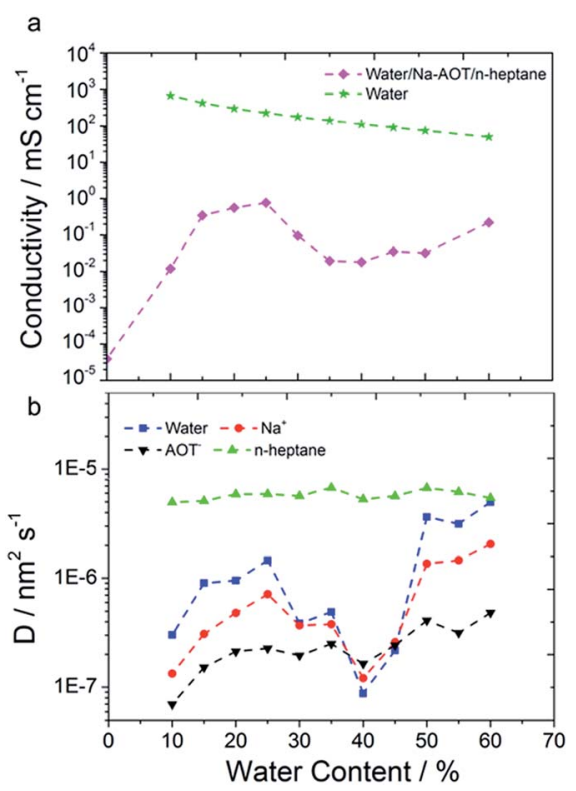

Fig. 7 (a) Conductivity as a function of water content measured experimentally by a conductivity meter (pink), and theoretically calculated assuming $\mathrm{Na}^{+}$as the only diffusing species contributing (green). (b) Diffusion coefficient for water, $\mathrm{Na}^{+}, \mathrm{AOT}$ and $n$-heptane calculated from the average MSD at $250 \mathrm{~ns}$. 
which the continuous phase is constituted by oil, the conductivity of which is nearly zero. All the water ("bound water") is immobilized in the core of the nanosized reverse micelles/ worms and participates in hydration of the surfactant head group associating with $\mathrm{Na}^{+}$ions and the head groups forming relatively rigid structures..$^{51,52}$ Therefore, in this system charge is transported by charged micelles and not by ions, resulting in a very low conductivity. ${ }^{51}$ When the water content is higher than required for the surfactant hydration, "free" water is available in the micelles. Upon addition of water, micelles grow in size and conductivity is slightly increased because of the higher mobility of the counter ions due to the charge hopping mechanism from one micelle to another. ${ }^{53}$ However, the conductivity is still below $10^{-4} \mathrm{mS} \mathrm{cm}^{-1}$.

With further increase of water content (10-30\%) the micelles increase in size, elongate and form cylindrical continuous structures which cause a significant jump in the conductivity due to better mobility of the ions in the continuous water channels compared to one in reverse micelles. When reverse micelles are formed again (35-50\%), the conductivity drops again. However, above $50 \%$ water content conductivity increases again due to continuous channel formation and above $70 \%$ water content significant increase of conductivity is observed due to phase inversion/separation. The increase in microemulsion conductivity observed when the water content is raised above the percolation threshold might result from a progressive aqueous droplet interlinking and clustering process. The results agree with the prediction of the geometrical model.

\section{Computational results}

MSD and diffusion coefficient investigation. We calculated $\mathrm{MSD}_{x}, \mathrm{MSD}_{y}$ and $\mathrm{MSD}_{z}$ for a representative sample of 100 beads for water, $\mathrm{Na}^{+}, n$-heptane and the AOT. Some examples of MSD curves calculated for $\mathrm{Na}^{+}$beads over $250 \mathrm{~ns}$ are reported in Fig. 8; all MSD curves for the other systems are reported in Fig. S18 and S19. $\dagger$ From the analysis of the curves and their behaviour as a function of time, it is possible to gain a deeper insight in the structure and continuity of the microemulsion. For example, Fig. 8a shows the MSD curves for the BME system c
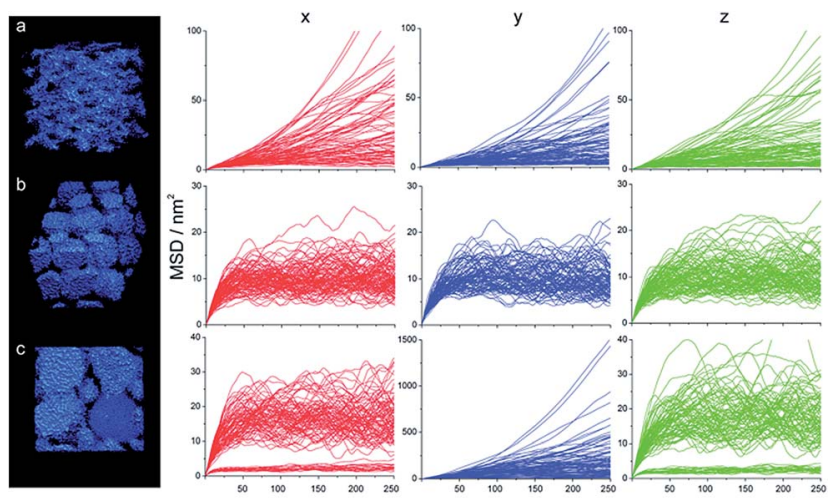

Time/ns

Fig. 8 MSD curves for $100 \mathrm{Na}^{+}$beads for the system containing (a) $20 \%$ (b) $40 \%$ (c) $50 \%$ water. The water structure is shown on the left. containing $20 \%$ water. MSD curves are similar in the three directions, implying it is an isotropic system. Additionally, part of the beads have a relatively limited mobility, being probably found in the neighbourhood of the surfactant head groups, as previously reported in systems with low water content. ${ }^{16}$ Some beads can however freely diffuse, as MSD curves keep growing as a function of time, corresponding to beads translating more than $10 \mathrm{~nm}$, basically all over the domain of the simulation cell, a cube of $10 \mathrm{~nm}$ side, Table 2. Fig. $8 \mathrm{~b}$ instead shows the MSD curves for the system $g$ containing $40 \%$ water. The system is isotropic as the curves are similar in the three directions. However, the MSD levels off very quickly. $\mathrm{Na}^{+}$beads are "caged" in spherical water pockets of a dimension corresponding to roughly $4 \mathrm{~nm}$ in inner diameter. Finally, Fig. 8c shows MSD curves for the system i containing $50 \%$ water.

The curves are different in the three directions. Ions can freely diffuse in the $y$ direction while they are "caged" in $x$ and $z$ directions. This is clearly shown also by the different scales, showing MSD up to $1500 \mathrm{~nm}^{2}$ in the $y$ direction and limited to $40 \mathrm{~nm}^{2}$ in $x$ and $z$ directions. In the $x$ and $z$ directions two populations are visible. This scenario corresponds to the coexistence of cylinders/worms of two different sizes, respectively roughly 4 and $1 \mathrm{~nm}$ in inner diameter.

Total MSD curves for all the species are reported in Fig. S20. $\dagger$ From the MSD curves, average diffusion coefficients were calculated according to the Einstein eqn (5) and these are plotted as a function of the water content in Fig. 7b. The oil diffusion coefficient does not significantly change as a function of water content. The diffusion coefficients of water, $\mathrm{Na}^{+}$and AOT beads instead undergo strong variations. As the water content varies, the hydrophilic species' diffusion is strongly influenced by the structure. Different structures can allow (essentially) free diffusion or induce caging effects as shown in Fig. 8. Diffusion is then higher for continuous structures and much lower for "caged" structures, such as the spherical micelles in system g. Since conductivity is due to diffusion of charged species, it is not surprising that $\mathrm{Na}^{+}$diffusion coefficient and conductivity behaviour as a function of water content are very similar, Fig. 7. However, $\mathrm{Na}^{+}$diffusion is much faster at higher water contents, e.g. $60 \%$, than at lower, e.g. $25 \%$, while for conductivity the trend is the opposite. This is due to the fact that at higher water contents, the concentrations of ions, even if more mobile, is significantly lower. The conductivity decreases then due to dilution, Fig. 7a. Conductivity strongly depends on the structure. This is also clear from the $60 \%$ simulations, systems $\mathrm{m}$ and $\mathrm{m}^{*}$. Two different structures were obtained, spherical micelles arranged in a FCC manner and cylinders, respectively. This might be due to a (i) long equilibration time required for the structure to stabilize, longer than $1 \mu \mathrm{s}$, (ii) kinetic trapping of the structure obtained in $\mathrm{m}$, and/or (iii) instability of the system. Indeed, in the section regarding SAXS experimental data, it will be shown that the $60 \%$ water content microemulsion structure varies over a time of 4 hours. This is mainly due to the much higher viscosity of the system, which has the appearance of a gel. MSD curves of the species for the two simulations are reported in Fig. S21. $\dagger$ AOT and $n$-heptane MSD only vary slightly for the two simulations, water and $\mathrm{Na}^{+}$ 
show instead a much higher mobility for the system $\mathrm{m}^{*}$, roughly 10 and 5 times higher on the $500 \mathrm{~ns}$ timescale, respectively. The diffusion coefficients were calculated in the same manner for the standard CG simulations. Fig. S22 $\uparrow$ compares the diffusion coefficients to the ones obtained for CG refined simulations. The results are very different and qualitatively in disagreement with experimental results.

The calculated values of diffusion coefficients cannot be used for quantitative analysis. It is well documented that time scales of dynamical processes in CG simulations need careful comparison to experimental data. ${ }^{25}$ It was observed in several studies that species in Martini CG simulations diffuse on average 4 times faster than in reality. ${ }^{25} \mathrm{AA}$ simulations provide in this case a much more reliable quantification of timedependent phenomena. In order to further investigate differences between CG and AA simulations, four equilibrated CG simulations were back mapped to AA and simulated for at least $50 \mathrm{~ns}$.

$A A$ vs. CG: morphology and MSD. The final snapshots of four CG simulations, corresponding to systems a, c, d, and $\mathrm{g}$ in Fig. 2, were back mapped to AA. Simulations were run for at least $50 \mathrm{~ns}$ in order to observe the stability of the structure. Table S3† summarizes the compositions, simulation time and size of the cell for the AA simulations. Fig. 9 reports the final structures obtained with AA simulations. Fig. 10a compares the MSD of the CG with the corresponding AA simulations.

For systems a and c, corresponding to 10 and $20 \%$ water content, respectively, structures obtained with AA and CG simulations are very similar, compare Fig. 6a and c to Fig. 9a and b, respectively. For the system d, containing $25 \%$ water, AA simulations resulted in partially interconnected structures, Fig. 9c, rather than cylinders obtained with CG simulations, Fig. 6d. According to the geometrical model, this composition

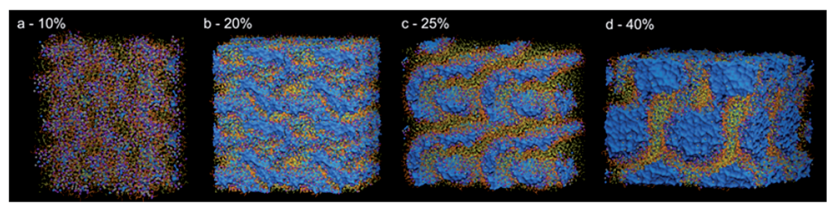

Fig. 9 AA simulation, final snapshot for system containing (a) $10 \%$, (b) $20 \%$, (c) $25 \%$ and (d) $40 \%$ water.
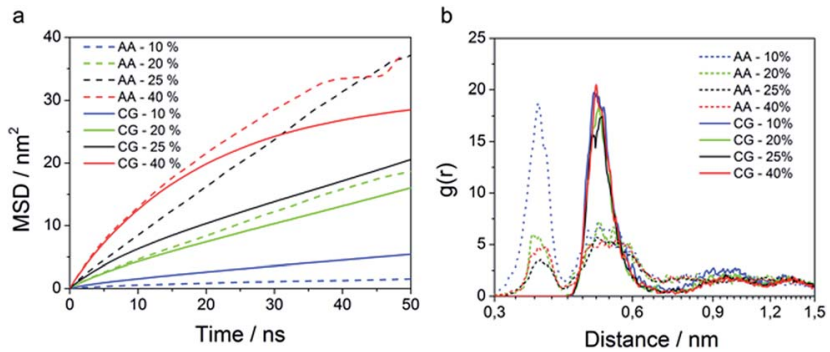

Fig. 10 Comparison of (a) the average MSD curves calculated for 100 $\mathrm{Na}^{+}$beads, and (b) $\mathrm{Na}^{+}-\mathrm{S}$ and $\mathrm{Na}-\mathrm{SO}_{3}$ RDFs for 10, 20, 25 and $40 \%$ water content with $A A$ (dashed lines) and CG (full lines) methods. lies at the boundary between two phases, BMEs and spherical micelles. It is possible that phase transitions might happen at slightly different concentrations in the CG and AA models, possibly due to different penetration abilities of water. Single water molecules in AA simulation can better penetrate in the surfactant film at the interface. Experimentally, it is difficult to discriminate between the two phases and thus assess which of AA or CG models better represents the system. The shape of water compartments could in principle be determined by angle resolved SAXS, but due to the macroscopic disorder of the phases (the structures are locally oriented but macroscopically they are isotropic) this measurement is not practicable. However, it is clear that both CG and AA structures are not bicontinuous. This means that the peak in the conductivity, Fig. 7a, that is commonly attributed to bicontinuous structures ${ }^{6}$ is obtained for structures that are not. Conductivity depends on how fast charges can travel in response to an electrical field. Most likely in cylindrical structures charges can travel faster in the straight channels than along a more tortuous route, such as the channels in BMEs. For system $g$ ( $40 \%$ water), both AA and CG simulations resulted in spherical micelles arranged in a FCC manner, Fig. 9d and 6g, respectively. In AA simulations however, interconnections between micelles can be observed.

Discrepancy between AA and CG simulation time-related quantities is a well known problem..$^{25}$ Motions in CG simulations tend to be generally faster, due to the lower amount of degrees of freedom resulting in reduced friction. However, different trends can be observed especially when simulating polar species.

Mobility can be compared only on very short time scales because AA simulations are prohibitively expensive to run for hundreds of ns for many systems. Average MSD curves for $\mathrm{Na}^{+}$ ions were calculated with AA simulations over $50 \mathrm{~ns}$, and compared to MSD curves for $\mathrm{Na}^{+}$beads calculated with CG simulations, Fig. 10a. AA MSD for the system containing $10 \%$ water is much lower than the one calculated by CG. In CG simulations, $\mathrm{Na}^{+}$is assumed to be fully hydrated, thus its motion is simultaneous with three associated water molecules. In reality, especially at low water content, $\mathrm{Na}^{+}$might not be fully hydrated, and especially can be in an intimate contact with the surfactant head group. In order to verify this, we calculate the RDF between $\mathrm{Na}^{+}$and surfactant head group (sulphur in atomistic) for both $\mathrm{AA}$ and $\mathrm{CG}$ simulations, Fig. 10b. In AA simulations, there is an additional peak at $0.36 \mathrm{~nm}$, much more pronounced for the $10 \%$ water content simulation than for any other system. This corresponds to $\mathrm{Na}^{+}$-ions that are in close contact with the surfactant head group. Obviously this distance cannot be captured by CG simulations, since the shorter distance is determined by the bead size, allowing closest approach to circa $0.5 \mathrm{~nm}$. For the system containing $20 \%$ water, very similar MSD curves are obtained: AA and CG structures are the same and the $\mathrm{Na}^{+}$is relatively often fully hydrated; thus in AA simulations $\mathrm{Na}^{+}$is more likely moving with its hydration water molecules. For the system containing $25 \%$ water, faster diffusion is obtained for AA, because of the occurrence of interconnected structures compared to cylindrical in CG. For the $40 \%$ simulations, slightly faster diffusion is obtained for AA 
simulations for the same reason, the presence of interconnections between water structures.

\section{Scattering}

Experimental results. SAXS results are presented in Fig. 11a. The general trend in the SAXS results is that the maximum in the scattering intensity shifts to larger $d$-spacing upon increasing the water content. For low water content $(20$ and $25 \%$ (data not shown)), an additional peak at around $2 \mathrm{~nm}$ can be noticed. This peak has been attributed to the surfactant molecules in previous work. ${ }^{3}$ The main peaks correspond to the size of water structure repeating units, and their positions were found to be in agreement with the size of the corresponding spherical/cylindrical micelles calculated with eqn (1) and (2) of the geometrical model, as shown in Fig. 11d. Interestingly for $50 \%$ water content, an additional peak appears at around $5 \mathrm{~nm}$. For $60 \%$ water content microemulsions, SAXS measurements averaged on 30 minutes were performed. The microemulsion resulted in a dynamic state during 4 hours, Fig. S23. $\dagger$ The observed $d$-spacing values were varying in number and in value with time. We observed an orientation of the sample respectively parallel and perpendicular to the capillary (min 150 and $\min 210$ ). The slow dynamics of these changes is due to the high viscosity of the microemulsion (more than $10 \mathrm{cP}$ ).

Dynamic light scattering measurements were also performed, and they are reported in Fig. 11c. It can be seen from Fig. 11c that upon increasing the water content, the size associated with the diffusion mode increases, and that from $20 \%$ on, two diffusion modes corresponding to two different sizes appear. The first one, around $1 \mathrm{~nm}$, was already found to correspond to the diffusion of the surfactant molecules, as found from SAXS measurements. As a demonstration of that, it
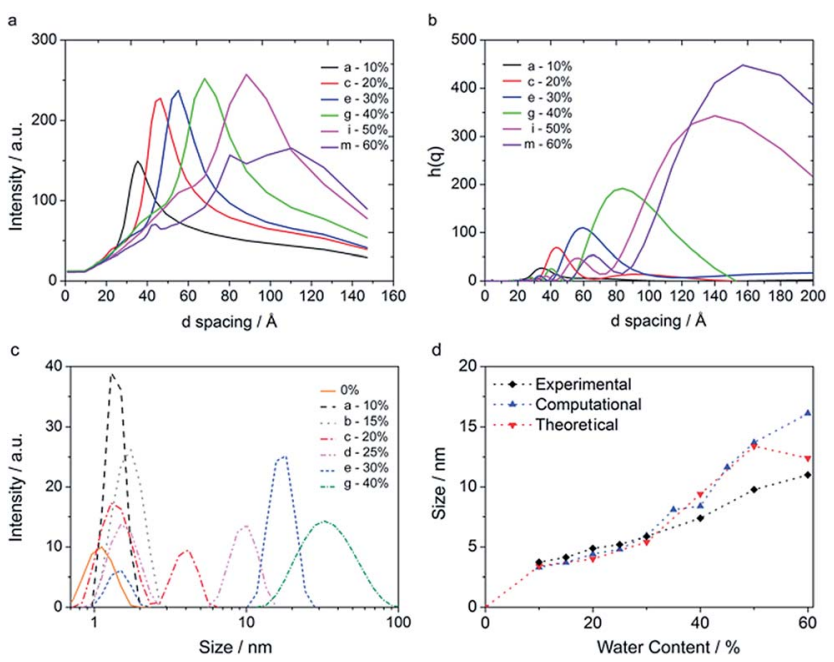

Fig. 11 (a) SAXS measurements performed for different water content microemulsions, (b) scattering function calculated from the endconfiguration of CG simulations, for the systems corresponding to the compositions a, c, e, g, i, and m depicted in Fig. 2, (c) DLS measurements as a function of water content (d) SAXS peaks compared to peak from the scattering function of simulations performed and theoretical values. can be seen from Fig. 11c that the intensity of it decreases as the water content and thus the "water aggregate" characteristic size increases. Additionally, the peak is already present when there is no water in the system, Fig. 11c. The second peak can be related to the dimension of water channels or micelles. However, the measured size is much bigger compared to the theoretical prediction, Fig. 11c. This can be due to the surfactant layer surrounding the water structure that contributes to the diffusion modes. Also, DLS may measure the motion of the interconnected channels, which is expected to be much lower than the single channel one.

Computational results. The scattering function $h(q)$ was calculated from the last frame of each simulation. For isotropic systems, peaks in $h(q)$ should correspond to peaks in the scattering intensity. We calculated the scattering function with a simplified approach, from the RDF of solely hydrophilic species, Fig. 11b, and with an exact method, taking into account the contribution of all the species. The results are hardly different, Fig. S24a. $\uparrow$ The major contributors to the scattering functions are indeed the electron-richer species, such as water, AOT head-group and $\mathrm{Na}^{+}$ion.

We investigated the effect of the box size on the scattering function calculated. We replicated the final equilibrated structure once in each direction, obtaining a final volume 8 times larger that the initial one. Compositions, size and details on this simulation are reported in Table S4. $\dagger$ For systems $\mathrm{g}$ and i, containing $40 \%$ and $50 \%$ water respectively, the system was also enlarged 8 times more, obtaining a final volume 64 larger than the initial one. Compositions, size and details on this simulation are reported in Table S5. $\dagger$ The size of the simulation box affected the calculated scattering function when the size of the box was similar to the size of the hydrophilic structure measured. For simulations containing more than $30 \%$ water, the smallest simulated system ( 800 AOT molecules) did not give a correct scattering response. No size could be detected, an example is reported in Fig. S25† for system g containing $40 \%$ water. When enlarging these systems 8 times by replicating the original system once in each direction, a $d$-spacing could be detected, which did not change when enlarging the volume to 64 times that of the original system, Fig. S24b and S25. $\dagger$ A summary of the scattering dimensions measured is reported in Fig. S24b $\dagger$ with different box sizes. Details of enlarged simulations are reported in Table S3 and S4. $\dagger$ Scattering functions calculated are reported in Fig. 11b. It must be noticed how both in the experimental and computational data, the system containing $50 \%$ water, i, shows two dimensions, 6 and $9 \mathrm{~nm}$ for the experimental and 6 and $13 \mathrm{~nm}$ for the simulation. This bimodality can be explained by the coexistence of water pockets of two different dimensions, as observed in Fig. 6i and 8c.

In general, computational and theoretical values obtained are in good agreement with experimental data for systems up to $40 \%$ water content. For systems containing more water, experimental values are a bit lower. This might be due to the fact that compositions approach phase separation, taking place at $65 \%$ water content. Because of their limited size, MD models have difficulties in correctly representing phase separation. The system might indeed rearrange in order to minimize the 
interfacial surface area to obtain the highest amount of "bulk water". In this case larger sizes would be obtained. Another possibility is that the real system has more "defects" and sizes are more polydisperse than in ideal conditions. This would lead to broader peaks, that we indeed observe, Fig. 11a.

\section{Conclusions}

We showed that CG Martini simulations, if correctly fine-tuned on the basis of atomistic structural data, can be used to map out phase diagrams of multiple-component systems. Phases simulated for Na-AOT- $n$-heptane-water systems are in agreement with theoretical studies and with experimental investigations. We showed that simple analysis tools can be used to better investigate the continuity of the phases. Bicontinuity was computationally visualized. In order to investigate the reliability of our model, we compared the diffusivity of $\mathrm{Na}^{+}$to experimental conductivity data and we show an exceptionally good agreement between the two. We back-mapped the equilibrium CG simulations to AA models and we were able to capture intimate ion-pair interactions between the $\mathrm{Na}^{+}$-ions and AOT anionic head group. Additionally, we calculated scattering functions that were found in good qualitative agreement with SAXS data, and quantitative agreement up to $40 \%$ water content. Disagreement at higher water contents was attributed to the fact that the systems were approaching phase separation that can not be simulated properly in such a small system.

CG modelling allows a tremendous reduction in amount of simulation time compared to atomistic models, e.g. for system $\mathrm{g}$ corresponding to $40 \%$ water content, CG simulation rate was of $622.5 \mathrm{~ns}$ per day while for AA simulation rate was $0.9 \mathrm{~ns}$ per day, more than 600 times slower. Similar time differences occur for equilibration of the simulation.

This work not only provides a full investigation of an important microemulsion system, but also is the basis for a "method" to simulate and compare this kind of systems to experimental data. We demonstrated that coarse graining allows for a reliable method to study realistic complex systems. It allows for a tremendous reduction of (atomistic) simulation and experimental time in particular when different compositions have to be screened in order to identify the optimal phase, for example for synthesis of nanoparticles or enhanced oil recovery.

\section{Acknowledgements}

We thank Ben Norder for SAXS measurements and discussions. We acknowledge financial support from the Advanced Dutch Energy Materials (ADEM) Program, the Ministry of Economic Affairs in the Netherlands in the framework of IOP-Self Healing Materials (SHM) Program and the COST CM1101 Action.

\section{Notes and references}

1 P. Winsor, Trans. Faraday Soc., 1948, 44, 376-398.

2 L. E. Scriven, Nature, 1976, 263, 123-125.
3 K. Kowlgi, U. Lafont, M. Rappolt and G. Koper, J. Colloid Interface Sci., 2012, 372, 16-23.

4 P. A. Reyes, J. A. Espinoza, M. E. Treviño, H. Saade and R. G. López, J. Nanomater., 2010, 948941.

5 E. Negro, R. Latsuzbaia and G. J. M. Koper, Langmuir, 2014, 30, 8300-8307.

6 A. Serrà, E. Gómez, G. Calderó, J. Esquena, C. Solans and E. Vallés, J. Electroanal. Chem., 2014, 720-721, 101-106.

7 W. Shang, X. Kang, H. Ning, J. Zhang, X. Zhang, Z. Wu, G. Mo, X. Xing and B. Han, Langmuir, 2013, 29, 13168-13174.

8 M. Subinya, A. K. Steudle, B. Nestl, B. Nebel, B. Hauer, C. Stubenrauch and S. Engelskirchen, Langmuir, 2014, 30, 2993-3000.

9 I. Moriguchi, R. Hidaka, H. Yamada, T. Kudo, H. Murakami and N. Nakashima, Adv. Mater., 2006, 18, 69-73.

10 J. G. E. M. Fraaije, K. Tandon, S. Jain, J.-W. Handgraaf and M. Buijse, Langmuir, 2013, 29, 2136-2151.

11 G. V. Mudzhikova and E. N. Brodskaya, Colloid J., 2006, 68, 729-737.

12 A. V. Nevidimov and V. F. Razumov, Mol. Phys., 2009, 107, 2169-2180.

13 A. V. Sangwai and R. Sureshkumar, Langmuir, 2011, 27, 6628-6638.

14 S. Abel, F. Sterpone, S. Bandyopadhyay and M. Marchi, J. Phys. Chem. B, 2004, 108, 19458-19466.

15 A. A. Bakulin, D. Cringus, P. A. Pieniazek, J. L. Skinner, T. L. C. Jansen and M. S. Pshenichnikov, J. Phys. Chem. B, 2013, 117, 15545-15558.

16 J. Chowdhary and B. M. Ladanyi, J. Phys. Chem. A, 2011, 115, 6306-6316.

17 D. De, M. Sajjan, J. Narayanan, J. R. Bellare and A. Datta, J. Phys. Chem. B, 2013, 117, 2106-2112.

18 J. Chowdhary and B. M. Ladanyi, J. Phys. Chem. B, 2009, 113, 15029-15039.

19 D. E. Moilanen, E. E. Fenn, D. Wong and M. D. Fayer, J. Am. Chem. Soc., 2009, 131, 8318-8328.

20 J. Faeder and B. M. Ladanyi, J. Phys. Chem. B, 2005, 109, 6732-6740.

21 J. Faeder and B. M. Ladanyi, J. Phys. Chem. B, 2000, 104, 1033-1046.

22 S. O. Nielsen, R. E. Bulo, P. B. Moore and B. Ensing, Phys. Chem. Chem. Phys., 2010, 12, 12401-12414.

23 P. Brocos, P. Mendoza-Espinosa, R. Castillo, J. Mas-Oliva and A. Pineiro, Soft Matter, 2012, 8, 9005-9014.

24 S. J. Marrink, A. H. de Vries and A. E. Mark, J. Phys. Chem. B, 2003, 108, 750-760.

25 S. J. Marrink and D. P. Tieleman, Chem. Soc. Rev., 2013, 42, 6801-6822.

26 W. Shinoda, D. E. Discher, M. L. Klein and S. M. Loverde, Soft Matter, 2013, 9, 11549-11556.

27 H. Lee and R. W. Pastor, J. Phys. Chem. B, 2011, 115, 78307837.

28 A. V. Martinez, L. Dominguez, E. Malolepsza, A. Moser, Z. Ziegler and J. E. Straub, J. Phys. Chem. B, 2013, 117, 7345-7351.

29 E. N. Brodskaya and G. V. Mudzhikova, Mol. Phys., 2006, 104, 3635-3643. 
30 P. Andre, B. W. Ninham and M. P. Pileni, New J. Chem., 2001, 25, 563-571.

31 J. N. Israelachvili, in Intermolecular and Surface Forces, ed. J. N. Israelachvili, Academic Press, Boston, Third edn, 2011, pp. 535-576.

32 W. Kunz, F. Testard and T. Zemb, Langmuir, 2008, 25, 112115.

33 B. Hess, C. Kutzner, D. van der Spoel and E. Lindahl, J. Chem. Theory Comput., 2008, 4, 435-447.

34 H. J. C. Berendsen, J. P. M. Postma, W. F. van Gunsteren, A. DiNola and J. R. Haak, J. Chem. Phys., 1984, 81, 3684-3690.

35 C. A. López, Z. Sovova, F. J. van Eerden, A. H. de Vries and S. J. Marrink, J. Chem. Theory Comput., 2013, 9, 1694-1708.

36 H. Lee, A. H. de Vries, S.-J. Marrink and R. W. Pastor, J. Phys. Chem. B, 2009, 113, 13186-13194.

37 C. Oostenbrink, A. Villa, A. E. Mark and W. F. Van Gunsteren, J. Comput. Chem., 2004, 25, 1656-1676.

38 H. J. C. Berendsen, J. P. M. Postma, W. F. van Gunsteren and J. Hermans, Intermol. Forces, 1981, 331-342.

39 B. Hess, H. Bekker, H. J. C. Berendsen and J. G. E. M. Fraaije, J. Comput. Chem., 1997, 18, 1463-1472.

40 S. Miyamoto and P. A. Kollman, J. Comput. Chem., 1992, 13, 952-962.

41 I. G. Tironi, R. Sperb, P. E. Smith and W. F. van Gunsteren, J. Chem. Phys., 1995, 102, 5451-5459.
42 A. J. Rzepiela, L. V. Schäfer, N. Goga, H. J. Risselada, A. H. De Vries and S. J. Marrink, J. Comput. Chem., 2010, 31, 13331343.

43 T. A. Wassenaar, K. Pluhackova, R. A. Böckmann, S. J. Marrink and D. P. Tieleman, J. Chem. Theory Comput., 2013, 10, 676-690.

44 W. Humphrey, A. Dalke and K. Schulten, J. Mol. Graphics, 1996, 14, 33-38, plates, 27-28.

45 D. S. Sivia, Elementary Scattering Theory: For X-ray and Neutron Users, OUP Oxford, 2011.

46 I. Lisiecki, P. André, A. Filankembo, C. Petit, J. Tanori, T. Gulik-Krzywicki, B. W. Ninham and M. P. Pileni, J. Phys. Chem. B, 1999, 103, 9176-9189.

47 R. Tanaka and T. Shiromizu, Langmuir, 2001, 17, 7995-8000. 48 S. J. Marrink, H. J. Risselada, S. Yefimov, D. P. Tieleman and A. H. de Vries, J. Phys. Chem. B, 2007, 111, 7812-7824.

49 V. R. Garigapati, J. Bian and M. F. Roberts, J. Colloid Interface Sci., 1995, 169, 486-492.

50 G. C. Shearman, O. Ces, R. H. Templer and J. M. Seddon, J. Phys.: Condens. Matter, 2006, 18, S1105.

51 Q. Li, T. Li and J. Wu, J. Colloid Interface Sci., 2001, 239, 522527.

52 G. X. Cheng, F. Shen, L. F. Yang, L. R. Ma, Y. Tang, K. D. Yao and P. C. Sun, Mater. Chem. Phys., 1998, 56, 97-101.

53 V. Arcoleo, M. Goffredi and V. T. Liveri, J. Solution Chem., 1995, 24, 1135-1142. 